\title{
Convergence of Migdal-Kadanoff Iterations in Non-Abelian Lattice Gauge Models
}

\author{
V. F. Müller and J. Schiemann \\ Fachbereich Physik, Universität Kaiserslautern, D-6750 Kaiserslautern, \\ Federal Republic of Germany
}

\begin{abstract}
We study the Migdal-Kadanoff recursion relations for lattice gauge models with gauge groups $\mathrm{SU}(N)$ or $\mathrm{U}(N)$ in dimensions $d<4$. It is shown that the Gibbs factor of a plaquette with Wilson action is driven to 1 for all values of the "temperature" (coupling constant). For models recently proposed by K.R. Ito, where Migdal's and Kadanoff's recursion relations hold exactly, a lower bound on the string tension is derived. The results obtained by us extend those derived by Ito for $\mathrm{U}(1)$. Our method is based on analytic continuation of the Gibbs factors, which are class functions, in the central angles.
\end{abstract}

\section{Introduction}

The recursion relations of Migdal [1] and the modified ones of Kadanoff [2] have been proposed as approximate real space renormalization group transformations both for spin systems and lattice gauge theories. However, only very recently these recursion relations have been investigated by purely analytic methods $[3,4]$. Ito [4] studied the $U(1)$ gauge group with Villain and Wilson action. For dimensions $d<4$ he showed that the effective actions generated by both types of recursion relations are always driven to the high temperature (strong coupling) region. Moreover he proposed special lattice gauge theory models in which the recursion relations of Migdal and of Kadanoff hold exactly and derived for $d=3$ a lower bound for the U(1) string tension of these models.

In this article we generalize Ito's work to the non-abelian gauge groups $\mathrm{SU}(N)$ and $\mathrm{U}(N)$. Migdal's $[\mathrm{M}]$ and Kadanoff's $[\mathrm{K}]$ recursion relations involve multiple convolutions of class functions on the gauge group $G, n \in \mathbb{N}_{0}$,

$$
\begin{aligned}
g^{(n+1)}(u) & =\left\{\frac{g^{(n) * r}(u)}{g^{(n) * r}(e)}\right\}^{q}, \\
g^{(n+1)}(u) & =\frac{\left(\left(g^{(n)}\right)^{q}\right)^{* r}(u)}{\left(\left(g^{(n)}\right)^{q}\right)^{* r}(e)},
\end{aligned}
$$

with $q, r$ positive integers, $u \in G$, and $e$ the unit element of $G$. 
Both recursion relations have the obvious fixed point $g(u) \equiv 1$. In Sect. 2 we define inductively analytic continuations in the central angles of the class functions entering both types of recursion relations. These analytic continuations are deduced from translations by elements of the maximal abelian subgroup in the recursion relations, the real parameters of which are then extended into the complex plane. This method avoids continuation of the Haar measure.

From these analytic properties we derive for gauge groups $\mathrm{SU}(N)$ or $\mathrm{U}(N)$ similar convergence properties of the effective actions and in Sect. 3 similar lower bounds for the string tensions as obtained by Ito in the case of U(1). Our main results are stated in Theorems 1 and 2 .

\section{Convergence of the Effective Actions}

We consider the gauge group $G=\mathrm{SU}(N)$ or $\mathrm{U}(N)$. The block plaquettes have size $l$ $\times l$ with $l=2,3,4, \ldots$, and we denote by $r$ the number of plaquettes in a block and by $q$ the number of identified block plaquettes,

$$
r=l^{2}, q=l^{d-2} \text {. }
$$

Let $g^{(n)}(u)$ be the Gibbs factor of a plaquette after $n$ renormalization group recursions, normalized at the unit element $e$ of $G$

$$
g^{(n)}(e)=1 \text {. }
$$

With $u_{j} \in G$ for $j=0,1,2, \ldots, r$, where $u_{0}=u$ and $u_{r}=e$, Migdal's and Kadanoff's recursion relations can be written in the following form $[4], n=1,2,3, \ldots$

$$
\begin{aligned}
& g^{(n)}(u)=\frac{1}{\mathscr{N}}\left\{\prod_{k=1}^{r-1} \int d u_{k} \prod_{j=1}^{r} g^{(n-1)}\left(u_{j-1} u_{j}^{*}\right)\right\}^{q}, \\
& g^{(n)}(u)=\frac{1}{\mathscr{N}^{\prime}} \prod_{k=1}^{r-1} \int d u_{k}\left\{\prod_{j=1}^{r} g^{(n-1)}\left(u_{j-1} u_{j}^{*}\right)\right\}^{q},
\end{aligned}
$$

with normalized Haar measure $d u$ and constants $\mathscr{N}, \mathcal{N}^{\prime}$ determined by the condition (2.2). In order to avoid clumsy notation we use the same symbols for the functions generated by both iteration schemes although they are not identical. Wilson's action is chosen on the original lattice, hence with $\beta \in \mathbb{R}_{+}$

$$
g^{(0)}(u)=e^{\beta \operatorname{Re}\{\operatorname{tr} u-N\}} .
$$

We first observe that the $g^{(n)}(u)$ are real positive and class functions on $G$, invariant under inversion of the argument

$$
\begin{gathered}
g^{(n)}\left(v u v^{-1}\right)=g^{(n)}(u), \forall v \in G, \\
g^{(n)}\left(u^{-1}\right)=g^{(n)}(u) .
\end{gathered}
$$

This is evident for (2.4) and follows then inductively from (2.3) due to invariance properties of the Haar measure.

Denote by $\left\{\lambda_{a}\right\}_{a \in K}$ a set of selfadjoint generators of the Lie-algebra of $G$ such that $A \subset K$ is the index subset corresponding to the maximal abelian subaigebra, i.e.,

$$
a, b \in A:\left[\lambda_{a}, \lambda_{b}\right]=0 .
$$


There are $M$ such generators, $M=N-1$, [N] in case of $\mathrm{SU}(N),[\mathrm{U}(N)]$. For $x \in \mathbb{R}^{M}, x=\left\{x_{a}\right\}_{a \in A}$, we define

$$
X=\sum_{a \in A} x_{a} \lambda_{a}
$$

and therewith particular group elements

$$
v_{j}=e^{-i\left(1-\frac{j}{r}\right) x}, \quad j=0,1, \ldots, r .
$$

Upon replacing $u_{j} \rightarrow v_{j} u_{j}$ in (2.3) we obtain, due to translation invariance of the Haar measure and (2.5), $n=1,2, \ldots$,

$$
\begin{aligned}
& g^{(n)}\left(e^{-i X} u\right)=\frac{1}{\mathscr{N}}\left\{\prod_{k=1}^{r-1} \int d u_{k} \prod_{j=1}^{r} g^{(n-1)}\left(e^{-i \frac{1}{r} X} u_{j-1} u_{j}^{*}\right)\right\}^{q}, \\
& g^{(n)}\left(e^{-i X} u\right)=\frac{1}{\mathscr{N}^{\prime}} \prod_{k=1}^{r-1} \int d u_{k}\left\{\prod_{j=1}^{r} g^{(n-1)}\left(e^{-i \frac{1}{r} X} u_{j-1} u_{j}^{*}\right)\right\}^{q} .
\end{aligned}
$$

For $g^{(0)}$ defined in (2.4), we write

$$
g^{(0)}\left(e^{-i X} u\right)=\exp \frac{1}{2} \beta\left\{\operatorname{tr}\left(e^{-i X} u\right)+\operatorname{tr}\left(u^{*} e^{i X}\right)-2 N\right\} .
$$

Equations (2.10) and (2.11) define for any fixed $u \in G$ real-valued functions of $x \in \mathbb{R}^{M}$,

$$
\tilde{g}^{(n)}(u, x):=g^{(n)}\left(e^{-i X} u\right)
$$

Moreover they define inductively analytic continuations of these functions by replacing

$$
X \rightarrow Z=X+i Y, x \rightarrow z=x+i y, \quad y \in \mathbb{R}^{M} .
$$

These analytic continuations form the basis of our treatment. We observe that the analytic continuations $\tilde{g}^{(n)}(u, z)$ have the form $g^{(n)}\left(e^{-i Z} u\right)$. This follows by uniqueness of the analytic continuation, starting from (2.3) with $u$ replaced by $e^{-i X} u$ and defining then inductively the analytic continuations.

Proposition 1. For $n \in \mathbb{N}_{0}$ the functions $\tilde{g}^{(n)}(u, z)$ are continuous in $u$ on $G$ for fixed $z \in \mathbb{C}^{M}$ and entire holomorphic in $z \in \mathbb{C}^{M}$ for any fixed $u \in G$.

Proof. Inspecting (2.11) the proposition is obvious for $n=0$. Assuming it to be true for $n-1$ we first conclude from the right-hand side of $(2.10)$ the continuity of $\tilde{g}^{(n)}$ in $u$ for fixed $z$, since only integrations of continuous functions on compact domains are involved.

In order to prove the proposed analytic properties it suffices to show [5] that for fixed $u \in G$ and any point $z \in \mathbb{C}^{M}, \tilde{g}^{(n)}$ is complex differentiable, i.e. holomorphic, in each $z_{a}, a \in A$, with the remaining $z_{b}, b \in A \backslash\{a\}$, kept fixed. Proceeding again inductively the properties of Proposition 1 for given $n-1$ allow in the analytically continued right-hand side of (2.10) to interchange differentiation with respect to $z_{a}$ and the group integration; this implies the analytic property for $n$. Compare [6] for a related theorem. The proof given there extends directly to our case. 
The class functions $g^{(n)}(u)$ are periodic functions of the central angles $\left\{\psi_{\alpha}\right\}_{\alpha=1,2, \ldots, M}: \forall u \in G \exists v \in G$ with

Hence

$$
\begin{gathered}
u=v \Delta v^{*}, \\
\Delta_{\alpha \beta}=\delta_{\alpha \beta} e^{-i \psi_{\alpha}}, \quad \alpha, \beta=1, \ldots, N, \\
\operatorname{SU}(N): \psi_{N}:=-\sum_{\alpha=1}^{N-1} \psi_{\alpha} .
\end{gathered}
$$

$$
g^{(n)}(u)=h^{(n)}\left(\left\{\psi_{\alpha}\right\}\right)
$$

We choose now such generators $\left\{\lambda_{a}\right\}_{a \in A}$ of the maximal abelian subalgebra, that the $\left\{x_{a}\right\}$ introduced in (2.8) are central angles. From Proposition 1, considered at $u=e$, together with (2.12) and (2.15) we obtain

Proposition 2. For $n \in \mathbb{N}_{0}$ the class functions $h^{(n)}\left(\left\{z_{a}\right\}\right)=\tilde{g}^{(n)}(e, z)$ are entire holomorphic in the analytically continued central angles $z_{a}=x_{a}+i y_{a}, a \in A$. $\tilde{g}^{(n)}$

We next derive upper bounds for these functions from bounds for the functions

From the analytically continued Eq. (2.11) follows easily

$$
\begin{gathered}
\left|\tilde{g}^{(0)}(u, i y)\right| \leqq g^{(0)}(u) \exp \left[\beta \sum_{\alpha=1}^{N}\left(\cosh y_{\alpha}-1\right)\right], \\
\mathrm{SU}(N): y_{N}=-\sum_{\alpha=1}^{M} y_{\alpha} .
\end{gathered}
$$

For real $t, 0<t<t_{0}$, we use

$$
\cosh t-1<B\left(t_{0}\right) t^{2}, t_{0}^{2} B\left(t_{0}\right)=\cosh t_{0}-1,
$$

and in the case of $\mathrm{SU}(N)$ in addition the inequality

$$
\left(\sum_{a=1}^{M} y_{a}\right)^{2} \leqq M \sum_{a=1}^{M} y_{a}^{2}
$$

Then introducing for $y \in \mathbb{R}^{M}$ the norm

$$
\|y\|=\max _{a \in A}\left|y_{a}\right|,
$$

we obtain for $\|y\|<\varrho$ :

$$
\left|\tilde{g}^{(0)}(u, i y)\right| \leqq g^{(0)}(u) \exp \left(\beta b_{e} \sum_{a=1}^{M} y_{a}^{2}\right),
$$

with the constant $b_{\varrho}$ defined by

$$
b_{\varrho}=\left\{\begin{array}{lll}
B(\varrho) & \text { for } & \mathrm{U}(N) \\
B(\varrho)+M B(M \varrho) & \text { for } & \mathrm{SU}(N) .
\end{array}\right.
$$

Proposition 3. With $b_{\varrho}$ of (2.20), for $n \in \mathbb{N}_{0}$ :

$$
\left|\tilde{g}^{(n)}(u, i y)\right| \leqq g^{(n)}(u) \exp \left[\beta b_{\varrho}\left(\frac{q}{r}\right)^{n} \sum_{a \in A} y_{a}^{2}\right] \quad \text { if } \quad\|y\|<\varrho r^{n} .
$$


Proof. Due to (2.19) the proposition is true for $n=0$. Employing this result in the right-hand side of the analytically continued Eqs. (2.10) and proceeding inductively concludes the proof.

From (2.21) we finally get bounds on the analytically continued class functions $h^{(n)}$; choosing in (2.21) $u=e^{-i X}$ we obtain

Proposition 4. For $n \in \mathbb{N}_{0}$ and $z=x+i y \in \mathbb{C}^{M}$ the entire holomorphic functions $h^{(n)}\left(\left\{z_{a}\right\}\right)$ are bounded for $\|y\|<\varrho r^{n}$ by

$$
\left|h^{(n)}\left(\left\{z_{a}\right\}\right)\right| \leqq h^{(n)}\left(\left\{x_{a}\right\}\right) \exp \left[\beta b_{\varrho}\left(\frac{q}{r}\right)^{n} \sum_{a \in A} y_{a}^{2}\right] .
$$

We consider now the Taylor expansion of the real function $h^{(n)}\left(\left\{x_{a}\right\}\right)$ with remainder of order 2. Due to (2.6) there are no linear terms, hence

$$
h^{(n)}\left(\left\{x_{a}\right\}\right)=1+\frac{1}{2 !} \sum_{k, l \in A} x_{k} x_{l}\left(\partial_{k} \partial_{l} h^{(n)}\right)\left(\left\{s x_{a}\right\}\right), 0<s<1 .
$$

Since the functions $h^{(n)}$ are holomorphic the derivatives in (2.23) have the Cauchy representation

$$
\left(1+\delta_{k l}\right)^{-1}\left(\partial_{k} \partial_{l} h^{(n)}\right)\left(\left\{s x_{a}\right\}\right)=\prod_{b \in A} \int_{C_{b}} \frac{d z_{b}}{2 \pi i} \frac{h^{(n)}\left(\left\{z_{b}\right\}\right)}{\prod_{a \in A}\left(z_{a}-s x_{a}\right)^{m_{a}}}, m_{a}=1+\delta_{a k}+\delta_{a l},
$$

with $C_{1} \times \ldots \times C_{M}$ chosen to be the distinguished boundary of a polydisc with center $\left\{s x_{a}\right\}$ and equal radii $R$. Together with (2.22) and the anticipated property $0<h^{(n)}\left(\left\{x_{a}\right\}\right) \leqq 1$, see (3.7), we obtain

$$
\left|\partial_{k} \partial_{l} h^{(n)}\left(\left\{s x_{a}\right\}\right)\right| \leqq\left(1+\delta_{k l}\right) \frac{\exp \left[\beta b_{\varrho}\left(\frac{q}{r}\right)^{n} M R^{2}\right]}{R^{2}}
$$

Minimizing this upper bound in $R$ gives

$$
\left|\partial_{k} \partial_{l} h^{(n)}\left(\left\{s x_{a}\right\}\right)\right| \leqq\left(1+\delta_{k l}\right) e \beta M b_{\varrho}\left(\frac{q}{r}\right)^{n}
$$

The application of $(2.22)$ requires

$$
\beta>\beta_{0}, \text { with } \frac{1}{\beta_{0} M}=\varrho^{2} b_{\varrho} .
$$

Collecting (2.23), (2.24), and inserting (2.1) we obtain our main result which we formulate in

Theorem 1. For $n \in \mathbb{N}_{0}$ the Gibbs factors $g^{(n)}(u)=h^{(n)}\left(\left\{x_{a}\right\}\right)$ satisfy on $G=\operatorname{SU}(N)$ or $\mathrm{U}(N)$ the uniform bounds

$$
\left|g^{(n)}(u)-1\right|<\sigma \beta l^{n(d-4)}, \quad \beta>\beta_{0} .
$$


The positive constant $\sigma$ does not depend on $\beta$ or $n$, however, it depends on the gauge group $G$ and on $\beta_{0}$.

Remark. For the abelian case $G=\mathrm{U}(1)$ the bound (2.26) has been derived by Ito [4]. It implies that for dimensions $d<4$ the effective actions generated by the recursion relations (2.3) are driven into the high temperature region with increasing $n$.

\section{String Tension}

Since the Gibbs factors $g^{(n)}$ are class functions, (2.5), they have the character expansion

$$
g^{(n)}(u)=\sum_{\tau \in \Gamma} d_{\tau} c_{\tau}^{(n)}(\beta) \chi_{\tau}(u), \quad n=0,1,2, \ldots
$$

where $\Gamma$ is the set of classes of inequivalent continuous irreducible unitary representations of $G, \chi_{\tau}$ the character of class $\tau$ and $d_{\tau}=\chi_{\tau}(e)$. The normalization (2.2) implies

$$
\sum_{\tau \in \Gamma} d_{\tau}^{2} c_{\tau}^{(n)}=1, \forall n \in \mathbb{N}_{0} .
$$

Proposition 5. For $n \in \mathbb{N}_{0}$ and $\forall \beta \in \mathbb{R}_{+}$and $\forall \tau \in \Gamma$ the coefficients $c_{\tau}^{(n)}(\beta)$ in the character expansion (3.1a) are real nonnegative in both cases $(2.3 \mathrm{M})$ and $(2.3 \mathrm{~K})$.

Proof. From the complete reducibility of all continuous finite-dimensional unitary representations of a compact group [10, Sects. (27.34) and (27.35)], follows $\forall \varrho, \sigma \in \Gamma$

$$
\chi_{\varrho}(u) \chi_{\sigma}(u)=\sum_{\tau \in \Gamma} m_{\tau}^{\varrho \sigma} \chi_{\tau}(u)
$$

with $m_{\tau}^{\varrho \sigma} \in \mathbb{N}_{0}$.

For $n=0$ we expand (2.4), denoting by $\varphi \in \Gamma$ the fundamental representation

$$
g^{(0)}(u)=e^{-\beta N} \sum_{m=0}^{\infty} \frac{1}{m !}\left(\frac{\beta}{2}\right)^{m}\left(\chi_{\varphi}(u)+\chi_{\bar{\varphi}}(u)\right)^{m} .
$$

Due to (3.2) the powers of characters appearing in the right-hand side yield linear combinations of characters with positive entire coefficients. Hence the claim is true in this case. We proceed inductively. Considering first Migdal's recursion relations $(2.3 \mathrm{M})$, we use the character expansion in the right-hand side and obtain

$$
g^{(n)}(u)=\frac{1}{\mathscr{N}}\left\{\sum_{\tau} d_{\tau}\left[c_{\tau}^{(n-1)}(\beta)\right]^{r} \chi_{\tau}(u)\right\}^{q}
$$

Since $q$ is a positive integer we can again use (3.2).

In the case of Kadanoff's recursion $(2.3 \mathrm{~K})$ we first observe that nonnegative expansion coefficients in $g^{(n)}(u)$ imply for the expansions

$$
\left(g^{(n)}(u)\right)^{q}=\sum_{\tau \in \Gamma} d_{\tau} b_{\tau}^{(n)}(\beta) \chi_{\tau}(u), \quad n=0,1,2, \ldots,
$$


nonnegative coefficients $b_{\tau}^{(n)}$, again because of (3.2). Moreover (2.2) yields

$$
1=\sum_{\tau \in \Gamma} d_{\tau}^{2} b_{\tau}^{(n)}(\beta), \forall n \in \mathbb{N}_{0} .
$$

Inserting (3.4) into the right-hand side of $(2.3 \mathrm{~K})$ gives

$$
g^{(n)}(u)=\frac{1}{\mathscr{N}^{\prime}} \sum_{\tau \in \Gamma} d_{\tau}\left[b_{\tau}^{(n-1)}(\beta)\right]^{r} \chi_{\tau}(u) .
$$

Hence we can proceed inductively.

As a corollary we obtain, due to (3.1b),

$$
0<g^{(n)}(u) \leqq 1, \quad n=0,1,2, \ldots .
$$

Remark. The functions $g^{(n)}(u)$ are continuous class functions of positive type. This can be deduced inductively: The convex cone of continuous class functions of positive type is closed under product and convolution, which easily follows from [10], Sects. (32.9) and (34.10) ("non-abelian Bochner's theorem"). Proposition 5 and (3.7) follow from these properties too.

Following Ito [4] we define

$$
\delta_{n}=\sum_{\substack{\tau \in \Gamma \\ \tau \neq 0}} d_{\tau}^{2} c_{\tau}^{(n)}(\beta)
$$

where $\tau=0$ denotes the trivial representation. Because of (3.1b)

$$
\delta_{n}=1-c_{0}^{(n)}=\int d u\left[1-g^{(n)}(u)\right] .
$$

Due to (3.7) we then have the following corollary of Theorem 1:

$$
0<\delta_{n}<\sigma \beta l^{n(d-4)} \text { for } \beta>\beta_{0} .
$$

For $\delta_{n}$ sufficiently small the character expansions can be used in both recursion relations (2.3) and lead to

Proposition 6. If $\delta_{n}<\frac{1}{2}$, then

$$
\delta_{n+1} \leqq\left(t \delta_{n}\right)^{r}
$$

with $t=2 q^{1 / r},\left[q \cdot 2^{q}\right]$ in the case of $\mathrm{M},[\mathrm{K}]$.

Proof. Starting with Migdal's recursion we get from (3.3)

$$
c_{0}^{(n+1)} \equiv \int d u\left\{\frac{\left(1-\delta_{n}\right)^{r}+\sum_{\tau \neq 0} d_{\tau}\left[c_{\tau}^{(n)}\right]^{r} \chi_{\tau}(u)}{\left(1-\delta_{n}\right)^{r}+\sum_{\tau \neq 0} d_{\tau}^{2}\left[c_{\tau}^{(n)}\right]^{r}}\right\}^{q} \geqq\left\{\frac{\left(1-\delta_{n}\right)^{r}}{\left(1-\delta_{n}\right)^{r}+\delta_{n}^{r}}\right\}^{q} .
$$

The result in the numerator follows from the positivity of the coefficients $c_{\tau}^{(n)}$ and (3.2). In the denominator we use

$$
\sum_{\tau \neq 0} d_{\tau}^{2}\left[c_{\tau}^{(n)}\right]^{r} \leqq\left[\sum_{\tau \neq 0} d_{\tau}^{2} c_{\tau}^{(n)}\right]^{r} .
$$


Hence, from (3.9) and (3.12)

$$
\delta_{n+1} \leqq 1-\left\{1+\left(\frac{\delta_{n}}{1-\delta_{n}}\right)^{r}\right\}^{-q}
$$

With $(1+x)^{-q}>1-q x$, for $x>0$, we obtain for $\delta_{n}<\frac{1}{2}$

$$
\delta_{n+1} \leqq q\left(\frac{\delta_{n}}{1-\delta_{n}}\right)^{r}<q\left(2 \delta_{n}\right)^{r}
$$

In Kadanoff's case we get by integrating (3.6),

$$
c_{0}^{(n+1)}=\frac{\left[b_{0}^{(n)}\right]^{r}}{\sum_{\tau \in \Gamma} d_{\tau}^{2}\left[b_{\tau}^{(n)}\right]^{r}} .
$$

Hence, due to (3.9)

$$
\delta_{n+1}=\frac{\sum_{\tau \neq 0} d_{\tau}^{2}\left[b_{\tau}^{(n)}\right]^{r}}{\left[b_{0}^{(n)}\right]^{r}+\sum_{\tau \neq 0} d_{\tau}^{2}\left[b_{\tau}^{(n)}\right]^{r}} \leqq\left(\frac{1-b_{0}^{(n)}}{b_{0}^{(n)}}\right)^{r} .
$$

This last inequality follows by neglecting the sum in the denominator and estimating the sum in the numerator as in (3.13). Moreover we used the normalization (3.5). From (3.1a), (3.4), and Proposition 5 we deduce with (3.2)

$$
b_{0}^{(n)}>\left[c_{0}^{(n)}\right]^{q}=\left(1-\delta_{n}\right)^{q} .
$$

Employing this in (3.15) gives

$$
\delta_{n+1}<\left\{\frac{1-\left(1-\delta_{n}\right)^{q}}{\left(1-\delta_{n}\right)^{q}}\right\}^{r} .
$$

For $\delta_{n}<1 / 2$ then follows

$$
\delta_{n+1}<\left(q \cdot 2^{q} \delta_{n}\right)^{r} .
$$

Obviously Proposition 6 is only useful if $t \delta_{n}<1$.

The final steps to derive a lower bound on the string tension in the case of $d<4$ are those of Ito's work [4]. For the reader's convenience we display them very briefly. Iterating (3.11) leads to

$$
n>n_{0}, \delta_{n}<\left(t^{\prime} \delta_{n_{0}}\right)^{r\left(n-n_{0}\right)}, t^{\prime}=t^{\frac{r}{r-1}} .
$$

Consider a series of quadratic loops $\mathscr{C}_{n}$ of size $l^{n} \times l^{n}$ forming after $n$ iterations the boundary of a plaquette respectively, and define the string tension $\alpha$ by

$$
\alpha=-\lim _{n \uparrow \infty} \frac{1}{l^{2 n}} \ln \frac{1}{d_{\varphi}}\left\langle\chi_{\varphi}\left(u_{\mathscr{C}_{n}}\right)\right\rangle .
$$

Due to Theorem 1 for each given $\beta$ there exists a convergent polymer expansion [7] for sufficiently large $n$, after iterating the renormalization group recursions $n$ 
times. $\mathscr{C}_{n}$ becomes the boundary of a plaquette, hence

$$
\frac{1}{d_{\varphi}}\left\langle\chi_{\varphi}\left(g_{\mathscr{C}_{n}}\right)\right\rangle=\frac{c_{\varphi}^{(n)}(\beta)}{c_{0}^{(n)}(\beta)}\left(1+\Delta_{n}\right) \leqq \frac{\delta_{n}}{1-\delta_{n}}\left(1+\Delta_{n}\right),
$$

where $\Delta_{n}$ is the higher order contribution in the infinite volume limit. $\Delta_{n}$ vanishes for $n \rightarrow \infty$. Using (3.18) with $t^{\prime} \delta_{n_{0}}<1$ in (3.20) implies

$$
\alpha>l^{-2 n_{0}} \ln \frac{1}{t^{\prime} \delta_{n_{0}}} .
$$

With $0<\varepsilon<1$ we define the function $v(\beta)$ and $n_{0}(\beta) \in \mathbb{N}_{0}$ by

$$
\begin{gathered}
t^{\prime} \sigma \beta l^{-v(\beta)(4-d)}=\varepsilon, \\
n_{0}(\beta)-1<v(\beta) \leqq n_{0}(\beta) .
\end{gathered}
$$

Hence, because of (3.10), we can deduce from inequality (3.21)

$$
\alpha>l^{-2 n_{0}(\beta)} \ln \frac{1}{\varepsilon} \geqq l^{-2(v(\beta)+1)} \ln \frac{1}{\varepsilon} .
$$

Inserting $l^{-2 v(\beta)}$ obtained from (3.22) we proved

Theorem 2. For gauge groups $\mathrm{SU}(N)$ or $\mathrm{U}(N)$, in the case of $d<4$ and $\beta>\beta_{0}$ the string tension $\alpha$ satisfies

$$
\alpha>\operatorname{const} \beta^{-\frac{2}{4-d}} \text {. }
$$

According to our derivation Migdal's or Kadanoff's recursion relations do not seem - for $d<4$ at least - to distinguish between abelian and non-abelian gauge groups.

The discrepancy between the bound (3.24) in case of $U(1)$ for $d=3$ and bounds derived within the full $\mathrm{U}(1)$-lattice gauge theory $[8,9]$ has already been pointed out by Ito. In spite of this deficiency the result derived for non-abelian gauge groups appears to us of some interest in view of the very difficult problem of spontaneous mass generation in $d=3$ non-abelian lattice gauge theories.

Acknowledgement. We thank the referee for pointing out an alternative proof of Proposition 5, outlined in the remark.

\section{References}

1. Migdal, A.A.: Recursion equations in gauge field theories. JETP 69, 810-822 (1975); Phase transitions in gauge and spin-lattice systems. JETP 69, 1457-1465 (1975)

2. Kadanoff, L.: Notes on Migdal's recursion formulas. Ann. Phys. 100, 359-394 (1976)

3. Collet, P., Eckmann, J.-P.: A spin glass model with random coupling. Commun. Math. Phys. 93, 379-406 (1984)

4. Ito, K.R.: Analytic study of the Migdal-Kadanoff recursion formula.Commun. Math.Phys.95, 247-255 (1984)

5. Hörmander, L.: An introduction to complex analysis in several variables, Sect. 2.2. Princeton, NJ: van Nostrand 1966 
6. Sansone, G., Gerretsen, J.: Lectures on the theory of functions of a complex variable, vol. I, Chap. 2.9.1. Groningen: Noordhoff 1960

7. See, for example, Seiler, E.: Gauge theories as a problem of constructive quantum field theory and statistical mechanics. In: Lecture Notes in Physics, Vol. 159. Berlin, Heidelberg, New York: Springer 1982

8. Göpfert, M., Mack, G.: Proof of confinement of static quarks in 3-dimensional U(1) lattice gauge theory for all values of the coupling constant. Commun. Math. Phys. 82, 545-606 (1982)

9. Ito, K.R.: Upper and lower bound for the string tension in the three-dimensional lattice quantum electrodynamics. Nucl. Phys. B 205 [FS5], 440-448 (1982)

10. Hewitt, E., Ross, K.A.: Abstract harmonic analysis. II. Berlin, Heidelberg, New York: Springer 1970

11. Bleher, P.M., Žalys, E.: Existence of long-range order in the Migdal recursion equations. Commun. Math. Phys. 67, 17-42 (1979)

Communicated by G. Mack

Received July 25, 1984; in revised form August 29, 1984

Note added in proof. After completing this work we became aware of [11], where the Migdal recursion equations for $\mathrm{U}(1)$ have been investigated by a different method in the case of $q>r$ in our notation. 